\title{
Multiscale Modeling of Phase Transformations in Steels
}

\author{
M. MILITZER, ${ }^{1,5}$ J.J. HOYT, ${ }^{2}$ N. PROVATAS, ${ }^{3}$ J. ROTTLER, ${ }^{4}$ \\ C.W. SINCLAIR, ${ }^{1}$ and H.S. ZUROB ${ }^{2}$
}

1.-Centre for Metallurgical Process Engineering, Department of Materials Engineering, The University of British Columbia, Vancouver, BC V6T 1Z4, Canada. 2.-Department of Materials Science and Engineering, McMaster University, Hamilton, ON L8S 4L8, Canada. 3.—Department of Physics, McGill University, Montreal, PQ H3A 2T8, Canada. 4.-Department of Physics and Astronomy, The University of British Columbia, Vancouver, BC V6T 1Z1, Canada. 5.-e-mail: matthias.militzer@ubc.ca

\begin{abstract}
Multiscale modeling tools have great potential to aid the development of new steels and processing routes. Currently, industrial process models are at least in part based on empirical material parameters to describe microstructure evolution and the resulting material properties. Modeling across different length and time scales is a promising approach to develop next-generation process models with enhanced predictive capabilities for the role of alloying elements. The status and challenges of this multiscale modeling approach are discussed for microstructure evolution in advanced low-carbon steels. Firstprinciple simulations of solute segregation to a grain boundary and an austenite-ferrite interface in iron confirm trends of important alloying elements (e.g., $\mathrm{Nb}, \mathrm{Mo}$, and $\mathrm{Mn}$ ) on grain growth, recrystallization, and phase transformation in steels. In particular, the linkage among atomistic simulations, phase-field modeling, and classic diffusion models is illustrated for the effects of solute drag on the austenite-to-ferrite transformation as observed in dedicated experimental studies for iron model alloys and commercial steels.
\end{abstract}

\section{INTRODUCTION}

Phase transformations during materials processing assume a crucial role in tailoring material properties for a wide variety of engineering applications, e.g., in the transportation, energy, and construction sectors. The austenite-ferrite transformation is a primary metallurgical tool to develop advanced steels with improved properties. For example, low-carbon advanced high-strength steels have microstructures with increased complexities (e.g., multiphase structures), resulting in properties of these materials that are crucial in the design of lightweight vehicles with improved fuel efficiency required to meet regulatory targets of reduced greenhouse gas emissions. ${ }^{1}$ Similarly, the formation of atomic-scale precipitates can be used to improve properties of steels and other alloys. For example, a new class of steels (NANO-HITEN) has been developed using the concept of interphase precipitation, i.e., the formation of fine carbides with microalloying elements $(\mathrm{V}, \mathrm{Nb}$, and $\mathrm{Ti})$ during the austenite-ferrite transformation, to maximize precipitation strength. ${ }^{2}$
To take advantage of the potential of these phase transformations it is critical to optimize processing paths for producing materials with the desired properties. Although significant progress has been made in developing knowledge-based process models for the steel industry, ${ }^{3-6}$ these models still require numerous empirical parameters that are typically determined from time-consuming laboratory experiments. Thus, these models are, strictly speaking, limited to the investigated steel chemistries and processing conditions. To increase the predictive capabilities of these models, it is imperative to formulate next-generation process models with a minimum of empirical parameters. In particular, it is sought to develop models that aid alloy design, which has become of increasing significance for advanced steels for which novel alloying concepts are being explored. Computational materials science now offers tremendous opportunities to formulate models containing fundamental information on the basic atomic mechanisms of microstructure evolution that can be implemented across different length and time scales. ${ }^{7-9}$ This approach essentially embraces the 
concept of integrated computational materials engineering (ICME) as a transformational discipline to efficiently develop advanced materials and their incorporation into the design of new products. ${ }^{10}$ ICME is considered to be a promising tool to accelerate innovation in the engineering of materials and manufactured products; i.e., it is critical for the steel industry to remain at the forefront of related research and development activities. By analyzing the physical mechanisms of microstructure evolution in general and that of phase transformations specifically, it is apparent that their kinetics is determined by interface migration rates that are markedly affected by the presence of alloying elements. For example, substitutional alloying elements (e.g., $\mathrm{Nb}$ ) can significantly retard recrystallization and the austenite-ferrite phase transformation in steels. Frequently, this retardation has been associated with a solute drag effect on the migrating interface thereby effectively reducing the interface mobility. Phenomenological solute drag models were proposed that introduce a binding energy and a trans-interface diffusivity to describe the solute-interface interaction. ${ }^{11-13}$ The atomistic mechanisms of the interaction of the solutes with moving interfaces are, however, still largely unexplored and have become an important research area. Mishin et al. ${ }^{9}$ recently reviewed atomistic modeling of interfaces and their impact on microstructure and properties citing 638 references.

Accurate modeling of the austenite [face-centered cubic (fcc)] to ferrite [body-centered cubic (bcc)] transformation in steels requires the free energy difference, i.e., driving pressure, between the two phases as a function of composition and temperature, as well as the effective mobility of the fcc-bcc interface. The driving pressure can be obtained from standard thermodynamic databases. The effective mobility is a kinetic parameter that is frequently employed as a fit parameter to replicate experimental transformation data. ${ }^{14,15}$ In a more rigorous way, the effective mobility can be constructed by combining the intrinsic mobility of the interface, i.e., the interface mobility in pure $\mathrm{Fe}$, with phenomenological solute drag models. ${ }^{16}$ In this approach, the two solute drag parameters, i.e., binding energy and trans-interface diffusivity, and the intrinsic mobility are employed as adjustable but physically well-defined parameters to replicate experimental observations of the austenite decomposition kinetics. Currently, intrinsic mobility and solute drag parameters cannot be determined exactly by independent experimental studies, but the tremendous increase in computational power during the past decades has made it realistic to use advances in computational techniques to quantify or, at least, to gain more insight into these parameters that describe atomistic events which determine the overall phase transformation as observed on the macroscale, i.e., the scale of a sample in the laboratory or a workpiece on an industrial line.
This article describes a multiscale modeling approach developed over the past 5 years for phase transformations in steels through a strategic research initiative in Canada as an example of similar research projects elsewhere in the world. The objectives of this research initiative are twofold:

1. Develop a new generation of industrial process models for the austenite-to-ferrite transformation kinetics in low-carbon steels that will be based on physically sound modeling approaches across different length scales to minimize the number of empirical parameters in the process model.

2. Validate individual models with experimental data on the laboratory scale and the overall process model with industrial data.

To achieve these objectives, the following computational and experimental research tasks have to be accomplished: (I) atomistic simulations using density functional theory (DFT), kinetic Monte Carlo (kMC), and molecular dynamics (MD); (II) phasefield simulations using phase-field crystal (PFC) and conventional mesoscale phase-field modeling (PFM); (III) model integration by deriving suitable phenomenological models based on atomistic and mesoscale simulations as well as dedicated laboratory simulations using model alloys; and (IV) validation of proposed models with experimental studies on ferrite formation in commercial steels. The status of the progress toward these four subtasks will be reviewed in this contribution.

\section{ATOMISTIC SIMULATIONS}

DFT is an ab initio atomistic simulation technique to obtain ground-state information, i.e., at $T=0 \mathrm{~K}$. In particular, the binding energies of solute atoms to interfaces and the activation energies of solute diffusion along and across interfaces can be determined with DFT. Here, DFT simulations have been performed using the commercial code VASP $^{17-19}$ that employs pseudo-potentials but permits to have calculation domains of 100-200 atoms that would be impractical with an all electron code (e.g., WIEN2k). First, binding and activation energies have been calculated for commonly used alloying elements $(\mathrm{Nb}, \mathrm{Mo}, \mathrm{Ti}, \mathrm{V}, \mathrm{Mn}, \mathrm{Ni}, \mathrm{Cr}, \mathrm{Co}, \mathrm{Si}$, and $\mathrm{Cu}$ ) at the $\Sigma 5$ (013) tilt grain boundary in bcc iron. ${ }^{20}$ This special grain boundary is selected as a model system for these simulations, as the high symmetry with a boundary unit cell of five atoms permits to establish a calculation domain with approximately 100 atoms by maintaining periodic boundary conditions. As illustrated in Fig. 1, the binding energy averaged over the five boundary sites increases with the size of solute atoms as the excess volume at the boundary permits to relieve the elastic strains associated with the solutes in the Fe matrix. Thus, a strong interaction of $\mathrm{Nb}, \mathrm{Mo}$, and $\mathrm{Ti}$, i.e., the larger solute atoms in $\mathrm{Fe}$, with the grain boundary is predicted 


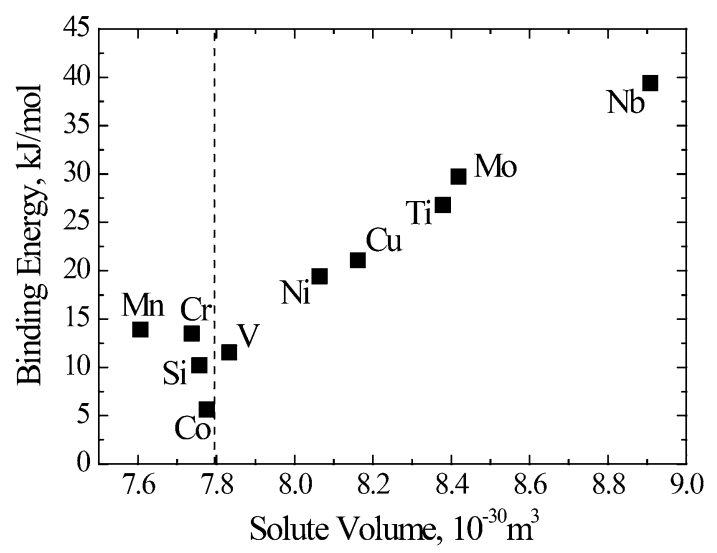

Fig. 1. Average binding energies to a $\Sigma 5$ grain boundary in bcc-Fe as calculated using DFT. The dashed line indicates the volume of the host $\mathrm{Fe}$ atoms.

with binding energies in the range of $25-40 \mathrm{~kJ} / \mathrm{mol}$. Also, some of the alloying elements (e.g., $\mathrm{Mn}, \mathrm{Cr}$, and $\mathrm{V}$ ) with an atom size similar to that of Fe do show significant binding energies of approximately $15 \mathrm{~kJ} / \mathrm{mol}$, which can be attributed to magnetic frustration, ${ }^{21}$ as these elements have antiferromagnetic interactions in an otherwise ferromagnetic system. Furthermore, the binding energy of $10 \mathrm{~kJ} / \mathrm{mol}$ for $\mathrm{Si}$ is in excellent agreement with experimental segregation studies for the $\Sigma 5$ grain boundary in an Fe-3 wt.\% Si bicrystal. ${ }^{22}$

In addition, the saddle point energies for different solute jumps along and across the $\Sigma 5$ boundary have been calculated with DFT to obtain information on the activation energy of solute grain boundary diffusion. Each of these jumps has a different activation barrier such that kMC simulations have to be performed; these different barriers are incorporated to determine the grain boundary diffusion coefficients. These simulations confirm grain boundaries as fast diffusion paths with activation energies that are $80-85 \%$ of those for lattice diffusion.

To extend DFT simulations to fcc-bcc interfaces, it is required to evaluate potential magnetic states of fcc Fe. At transformation temperatures (e.g., $900^{\circ} \mathrm{C}$ ), fcc $\mathrm{Fe}$ is paramagnetic, but for the groundstate DFT calculations ( $T=0 \mathrm{~K}$ ), one must find a suitable magnetic approximation for fcc Fe with a total magnetization of zero. After careful considerations, an antiferromagnetic double layer (AFMD) structure is assumed for fcc Fe, and solute binding energies have been determined at a selected fcc-bcc interface with a Kurdjumov-Sachs orientation relationship that is frequently observed for the austenite-ferrite transformation. These simulations confirm that $\mathrm{Nb}$ and Mo have, as the larger solutes, the largest binding energies. The binding energies for $\mathrm{Nb}$ and Mo are predicted to be approximately $25 \mathrm{~kJ} / \mathrm{mol}$ and $15 \mathrm{~kJ} / \mathrm{mol}$, respectively, whereas for $\mathrm{Mn}$ this value is approximately $10 \mathrm{~kJ} / \mathrm{mol}$. These findings are consistent with experimental observations of the strong effects of $\mathrm{Nb}$ and Mo on delaying the austenite-to-ferrite transformation in lowcarbon steels. Furthermore, it is remarkable to note the similarity of these values with those predicted for the $\Sigma 5$ grain boundary, suggesting that in particular the strong segregation of the larger solutes can be explained based on the excess volume at boundaries and interfaces. Thus, excess volume can be taken as an indicator to evaluate whether the binding energies obtained for the investigated special interfaces remain of merit for more general grain boundaries and interfaces. A systematic MD study for more general grain boundaries (e.g., $\Sigma 29$ and $\Sigma 61$ ) in bcc Fe indicates that the average excess volume of these boundaries is comparable to that of the $\Sigma 5$ boundary. ${ }^{20}$ Based on these encouraging results, an attempt can now be made to investigate with DFT the concurrent interaction of different alloying elements with the interfaces. Some initial results for the $\Sigma 5$ boundary indicate the potential complexity of these interactions. For example, an increase of the binding energy by up to $30 \%$ is predicted when larger solutes (e.g., $\mathrm{Nb}-\mathrm{Mo}$ and $\mathrm{Nb}-\mathrm{Ti}$ ) are placed together at intermediately spaced boundary sites (e.g., $0.36 \mathrm{~nm}$ apart). ${ }^{20}$ It will be crucial to explore these interactions with atomistic simulations such that trend predictions can be made for commercial steel chemistries that typically contain a multiplicity of alloying additions.

Classic MD simulations using an embedded atom method (EAM) potential for pure Fe can be used to obtain information on the intrinsic mobility of the fcc-bcc interface. ${ }^{23}$ Here, simulation cells have been employed with periodic boundary conditions in all directions and utilizing a bcc-fcc-bcc sandwich structure containing roughly 55,000 atoms. A lowindex orientation of the fcc-bcc interface was chosen, i.e., (110) bcc//(111) fcc and [001] bcc//[1-10] fcc. But this interface is slightly tilted by $5.26^{\circ}$ to create a series of steps at the interface. The steps allow for sufficient mobility of the interface. From the interface velocity and the computed free energy difference between the fcc and bcc phases, a mobility can be extracted. Figure 2 shows an Arrhenius plot of mobility values as obtained for different temperatures, $T$. Clearly, the mobility follows, as expected, an Arrhenius behavior, i.e.,

$$
M=M_{0} \exp (-Q / R T)
$$

where $M_{0}$ is the pre-exponential factor, $Q$ is the activation energy, and $R$ is the ideal gas constant. An activation energy of $16 \mathrm{~kJ} / \mathrm{mol}$ is concluded from the MD simulations, which is about one order of magnitude lower of what has conventionally been concluded from experimental data, i.e., $140 \mathrm{~kJ} /$ mol. ${ }^{24}$ The calculated mobility values may reflect a particular transformation situation (e.g., at the tip of a Widmanstätten side plate) but may not be applicable to the case of motion of a planar ferrite interface. Furthermore, the mobility does in general depend on the crystallographic orientation, and 


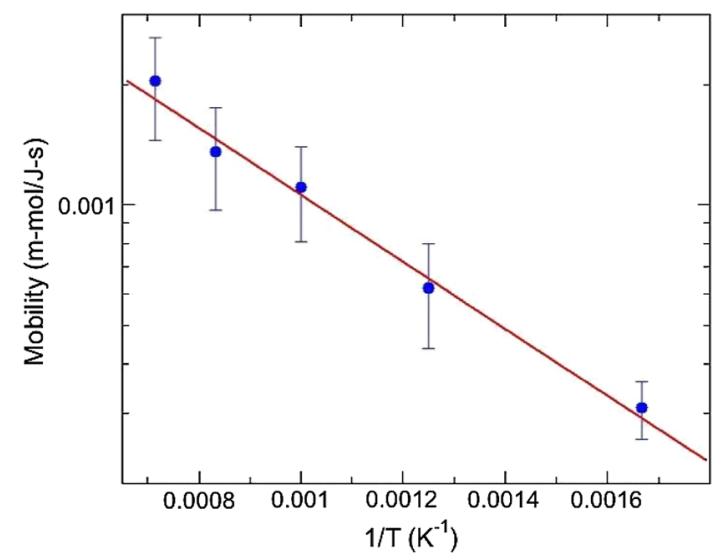

Fig. 2. Arrhenius plot of the intrinsic mobility as obtained by MD simulations for the selected low-index fcc-bcc interface. ${ }^{23}$

future studies will be required to determine these dependencies in detail.

Nevertheless, the current MD simulations are able to identify the atomic mechanisms taking place during the migration of the selected fcc-bcc interface. ${ }^{25}$ A centrosymmetry parameter (CSP) has been used to discriminate between atoms of the fcc and bcc phases. It is shown from both simulation and disconnection theory that the primary structural disconnections formed when creating the fcc-bcc bicrystal do not move laterally across the boundary as the transformation proceeds. However, it is observed that a second set of glissile disconnections forms on the terraces and the interface migrates by the rapid advance of these mobile defects. The ratelimiting step of the interface propagation process is the nucleation of new bcc islands on the terraces, and it is shown that the nucleation event is heterogeneous, with the primary disconnections acting as the preferred nucleation sites, as illustrated in Fig. 3. The nucleation and growth mechanisms identified in this article may provide important insights into the mobility of more general incoherent interphase boundaries.

\section{PHASE-FIELD MODELING}

Over the past two decades, the PFM approach has been increasingly used as a mesoscale modeling tool to simulate microstructure evolution. Initially, PFMs were developed for solidification, but more recently they have also been applied to solid-solid phase transformations in steels. ${ }^{26}$ In particular, the austenite-to-ferrite transformation in steels has been widely described with phase-field models. ${ }^{14,15,27}$ The solute drag effect has been incorporated into phase-field models. ${ }^{28,29}$ In particular, a recently developed friction force approach efficiently accounts for the role of the atomistic-scale soluteinterface interaction in microstructure evolution simulations. ${ }^{29}$ Furthermore, the PFC method has been examined as a potential modeling technique to bridge the gap between the coarse-grained phase-field

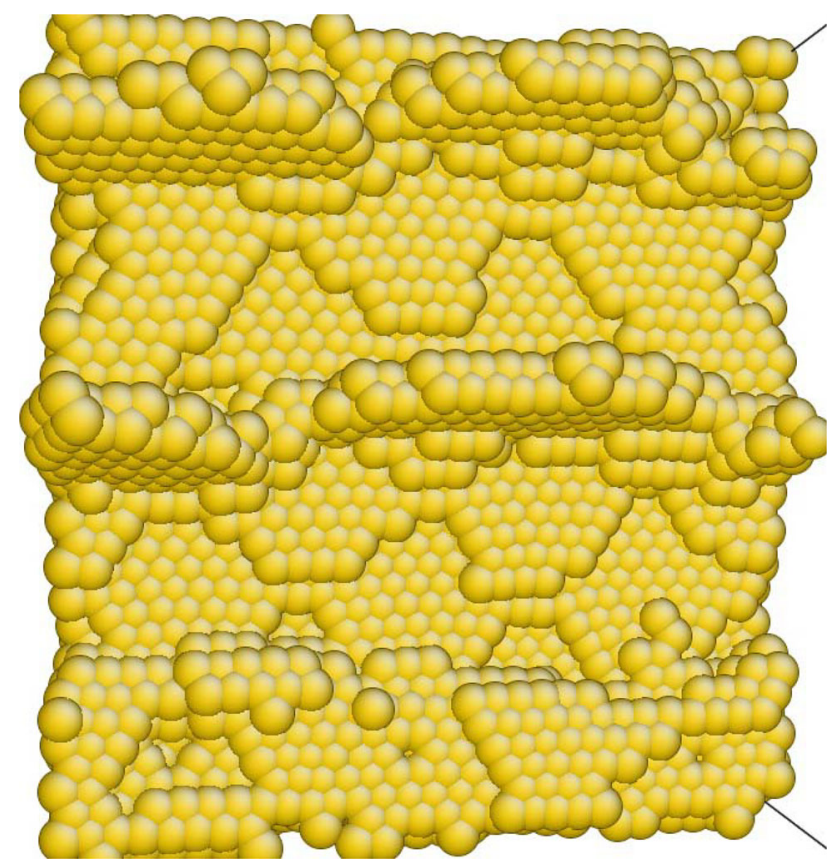

Fig. 3. Normal view of the migrating fcc-bcc interphase boundary in MD simulations. Only the bcc atoms are shown. The migration mechanism is the nucleation of mobile steps on the terraces between the primary steps. ${ }^{25}$

techniques and atomistic MD simulations. The PFC model maintains the atomistic resolution of the MD simulations but operates on the mesoscopic (i.e., diffusional) time scales of the phase-field model. Thus, the PFC modeling paradigm is emerging as the model of choice when investigating materials phenomena with atomistic-scale effects over diffusive time scales. Recent variants of the PFC model, so-called structural PFC (XPFC) models introduced by Greenwood et al. ${ }^{30,31}$, have further increased the capability of the method by allowing for easy access to various structural transformations. In particular, PFC simulations have been performed to explicitly track solute drag on the atomistic scale. ${ }^{32}$ These simulations confirm that the solute atoms slow down the grain boundary migration that occurs by a stopand-go mechanism, which is particularly prominent for strong solute drag (i.e., for lower driving pressures where the solute enrichment can move with the interface). For larger driving pressure, a clear transient stage is observed where the interface gradually loses the solute spike and moves subsequently similar to an intrinsic boundary without any solutes (or negligible effects of solutes on boundary motion). The simulation results are, in a first approximation, consistent with classical phenomenological solute drag theories thereby providing a further justification of their use in phenomenological transformation models. Some comparatively minor differences in the solute drag modeling can be attributed primarily to the role of the boundary structure that is explicitly considered in PFC simulations but not in the phenomenological theories. 
In addition, the phase-field approach offers the tremendous opportunity for a direct linkage of PFC and PFM. The PFC-PFM integration would constitute a breakthrough in developing a truly multiscale model by providing a seamless model approach that directly accounts for the role of atomistic-scale phenomena on microstructure evolution. As an important step toward the proposed model integration, an amplitude expansion of the PFC models has been developed leading to a mesoscale complex orderparameter (amplitude), i.e., a phase-field representation, model for, at this stage, two-dimensional square-triangular structures. ${ }^{33}$ Amplitude models retain the salient atomic-scale features of the underlying PFC model while resolving microstructures on mesoscales as in coarse-grained PFMs. The applicability and capability of this complex amplitude model has been demonstrated with simulations of peritectic solidification and grain growth exhibiting the emergence of secondary phase structures. An application to the austenite-ferrite transformation has yet to be accomplished and will require to extend the amplitude expansion approach to three-dimensional bcc-fcc structures.

\section{FERRITE GROWTH IN FE MODEL ALLOYS}

An important aspect of the development of models is their validation with experiments. As a first step of this validation, systematic laboratory experiments were conducted using Fe model alloys where ferrite growth was monitored using the method of controlled decarburization. ${ }^{34,35}$ These experiments consist of reheating the specimen into the austenite single-phase region in an atmosphere of wet hydrogen. The oxygen potential of this atmosphere is high enough to remove carbon from the specimen but too low to oxidize iron. The removal of carbon from the specimen is controlled by diffusion in the solid and leads to the formation of ferrite in regions with lower carbon levels. If the specimen is sufficiently thick, then an overlap of the carbon diffusion profiles does not take place, resulting in a simple one-dimensional diffusion problem and the emergence of a planar interface between ferrite and austenite (see Fig. 4). The simplicity of the growth geometry along with the availability of accurate thermodynamic and kinetic databases allows one to use these experiments to deduce the contact conditions at the migrating ferrite-austenite interface and to determine the magnitude of solute drag in Fe-C- $X$ model alloys $\left(X: \mathrm{Ni}, \mathrm{Mn}, \mathrm{Si}, \mathrm{Mo}\right.$, etc.). ${ }^{35} \mathrm{~A}$ self-consistent model has been developed to describe the observed ferrite growth. ${ }^{36}$ The model captures the time evolution of interfacial contact conditions for substitutional and interstitial solutes. Substitutional element solute drag is evaluated in terms of the dissipation of free energy within the interface, and an estimate is provided for the rate of buildup of the alloying element "spike" in austenite. The transport of the alloying elements within the

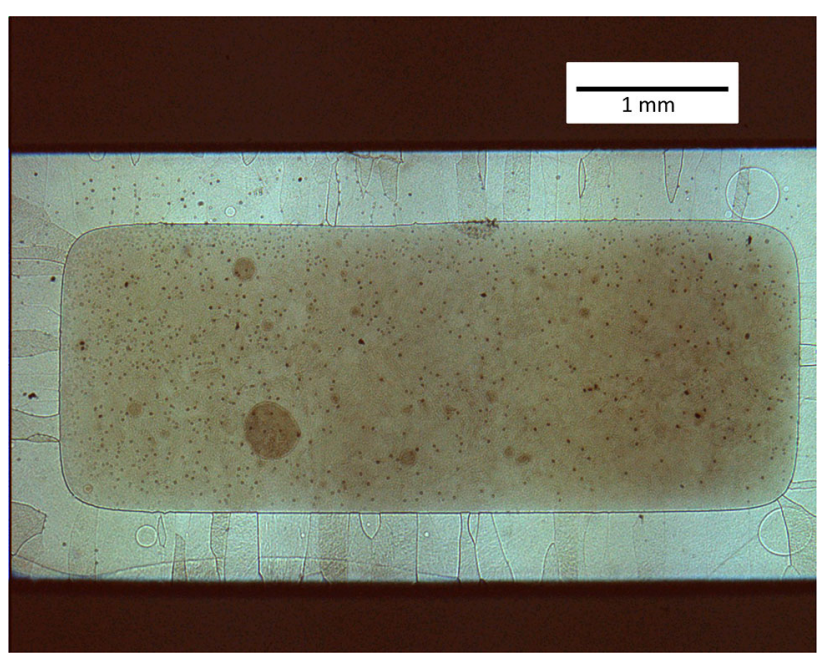

Fig. 4. Typical micrograph of decarburization tests showing ferrite (white) on the sample surface and austenite (dark) in the inside of the sample.

interface region is described using a discrete-jump model. For each investigated ternary system, the values of the cross-boundary diffusion coefficient and the solute-interface binding energy have been obtained. The binding energies concluded from this analysis are with $15 \mathrm{~kJ} / \mathrm{mol}$ for Mo, $9 \mathrm{~kJ} / \mathrm{mol}$ for Si, and below $5 \mathrm{~kJ} / \mathrm{mol}$ for $\mathrm{Mn}$; $\mathrm{Cr}$ and Ni qualitatively consistent with the trends predicted by the DFT simulations. Experimental measurements of the solute binding energy to the interface are presently underway using the three-dimensional atom-probe technique. The initial results indicate substantial enrichment of Mo and $\mathrm{Cr}$ but not of $\mathrm{Si}$ and $\mathrm{Ni}$ at the investigated interfaces. The atom-probe results have also revealed the strong segregation of $\mathrm{C}$ to the interface. This strong interaction has been included neither in the DFT simulations nor in the ferrite growth model to describe the decarburization experiments. The incorporation of the interaction between carbon and the substitutional solute atoms at the interface is expected to modify the aforementioned binding energies, particularly in the case of elements that strongly interact with C. Furthermore, the decarburization studies have recently been extended to the Fe-C-Mn-Si system indicating that solute drag of $\mathrm{Mn}$ and Si can be added without considering any interaction between these two alloying elements, which are rather weakly segregating elements. Based on the DFT studies, one may, however, expect more complex interactions between stronger segregating alloying additions, e.g., Mo and Nb.

\section{MODEL APPLICATION TO COMMERCIAL STEELS}

The Johnson-Mehl-Avrami-Kolmogorov (JMAK) model is frequently employed by adopting the additivity principle to describe the transformation 
kinetics in microstructure process models. ${ }^{6}$ For isothermal transformations, the JMAK model is given by

$$
X=1-\exp \left(-b t^{n}\right)
$$

where $X$ is the fraction transformed, $t$ is time, and $b$ and $n$ are parameters fitted to experimental data. For the austenite-to-ferrite transformation, $n=1$ and $b$ depends on temperature as well as on steel chemistry and austenite microstructure. The available austenite-ferrite transformation data for isothermal and continuous-cooling tests (CCT) have been reanalyzed to facilitate the interpretation of the fit parameter $b$ in terms of physically relevant parameters, i.e., the intrinsic mobility (see Eq. 1) and the solute drag parameters consisting of the binding energy $E$ and the trans-interface diffusivity $D$, which can be expressed as

$$
\frac{D}{\delta}=\frac{D_{0}}{\delta} \exp \left(-Q_{\mathrm{b}} / R T\right)
$$

where $\delta$ is the interface width (usually assumed to be $1 \mathrm{~nm}$ ), $D_{0}$ is the pre-exponential term, and $Q_{\mathrm{b}}$ the activation energy for boundary diffusion. The preexponential term can be inferred from bulk diffusion, e.g., by using the geometric mean of the preexponential factors for lattice diffusion in bcc and fcc, respectively. Then, $Q_{\mathrm{b}}$ and $E$ are employed together with the intrinsic mobility as adjustable parameters to replicate the overall austenite -to-ferrite transformation kinetics.

First, one may consider this approach for ferrite formation in binary $\mathrm{Fe}-\mathrm{Mn}$ alloys including an interstitial-free (IF) steel that for the transformation analysis can be approximated as an $\mathrm{Fe}$ 0.1 wt.\% Mn alloy. Here, there is no carbon in solution such that the transformation kinetics is interface controlled, thereby simplifying the analysis significantly compared to the more general case of low-carbon steels, where the long-range diffusion of carbon has to be considered such that the reaction becomes mixed mode. For the Fe-Mn system, adopting the conventional activation energy of $140 \mathrm{~kJ} / \mathrm{mol}$ for the intrinsic mobility enables a consistent description of the phase transformation kinetics in alloys with different Mn concentrations (up to 2 wt.\%). ${ }^{37}$ Figure 5 compares the model with the CCT behavior in the IF steel. The activation energy for trans-interface diffusion is $94 \%$ of the value for $\mathrm{Mn}$ bulk diffusion in bcc-Fe, and the binding energy for $\mathrm{Mn}$ to the austenite-ferrite interface decreases from $18 \mathrm{~kJ} / \mathrm{mol}$ to $6 \mathrm{~kJ} / \mathrm{mol}$ when the $\mathrm{Mn}$ content increases from 0.1 wt.\% to $2 \mathrm{wt} . \%$. These binding energies agree well with the predictions of the DFT simulations $(10 \mathrm{~kJ} / \mathrm{mol})$, the decarburization studies, and limited measurements of $\mathrm{Mn}$ grain boundary segregation $(5-10 \mathrm{~kJ} /$ mol). ${ }^{38,39}$ The effect of Mn content on the apparent binding energy can be rationalized with different binding energies to individual sites in the interface

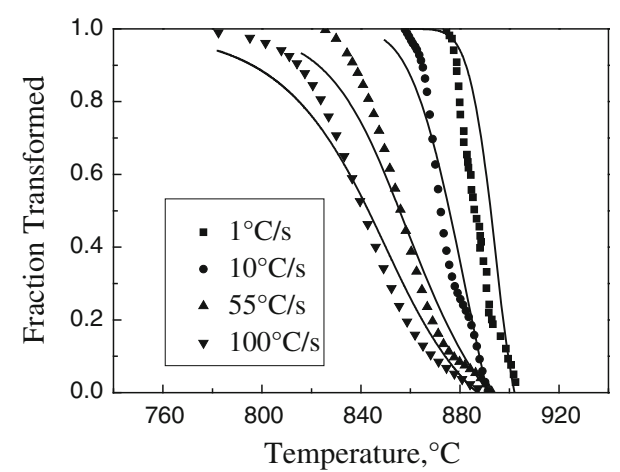

Fig. 5. Comparison of experimental CCT data (symbols) with solute drag model calculations (lines) for an IF steel (Fe-0.1\%Mn). ${ }^{37}$

region, as predicted by DFT. As the Mn content increases, so does $\mathrm{Mn}$ segregation to the interface, i.e., $\mathrm{Mn}$ atoms have to occupy increasingly less favourable sites leading to a decrease in the apparent binding energy.

Furthermore, the effect of $\mathrm{Nb}$ in solution on the austenite-to-ferrite transformation kinetics in an X65 linepipe steel microalloyed with $0.047 \mathrm{wt} . \% \mathrm{Nb}$ can be described with a mixed-mode model in which long-range carbon diffusion in austenite is combined with the interface reaction that includes solute drag. ${ }^{16}$ This model approach permits to quantitatively describe the formation of ferrite as a function of cooling rate and the amount of $\mathrm{Nb}$ in solution. As in the Fe-Mn case, $140 \mathrm{~kJ} / \mathrm{mol}$ is adopted for the activation energy of the intrinsic interface mobility. A binding energy of $48 \mathrm{~kJ} / \mathrm{mol}$ and an activation energy for the trans-interface diffusion of $263 \mathrm{~kJ} /$ mol is concluded to capture the $\mathrm{Nb}$ solute drag effect on the migrating fcc-bcc interface. The value for the binding energy is in reasonable agreement with the DFT predictions. The trans-interface diffusion coefficient falls in between those reported for bulk diffusion of $\mathrm{Nb}$ in fcc and bcc iron, respectively, supporting the assumption to approximate its value by the geometric mean of bulk diffusion in austenite and ferrite as proposed based on the decarburization studies. ${ }^{36}$

The current analysis provides evidence that, at least for steel chemistries for which the austeniteferrite transformation kinetics is predominantly affected by one substitutional alloying element, trend predictions for solute drag can be made with binding energies obtained from DFT simulations and trans-interface diffusion coefficients inferred from available data on bulk diffusion in bcc and fcc iron. There are, however, still, a number of limited experimental studies (e.g., CCT tests) required to fine tune the solute drag parameters for sufficiently accurate transformation models with predictive capabilities. For steels with extra-low- and lowcarbon contents, the intrinsic mobility is another parameter that is needed for the transformation model. Even though an activation energy of 
$140 \mathrm{~kJ} / \mathrm{mol}$ seems to be an established value, there is a great deal of uncertainty about the pre-exponential term, i.e., $M_{0}$, that remains at this stage a truly empirical parameter. For example, the $M_{0}$ values concluded for the IF steel and the linepipe steel, respectively, differ by two orders of magnitude. Further investigations are required to rationalize this apparent chemistry dependence of the mobility.

\section{CONCLUSION}

The proposed multiscale modeling approach shows significant promise for developing austenite decomposition models with a greatly reduced number of fitting parameters. In particular, the combination of atomistic simulations with dedicated transformation studies on model systems will allow the more reliable prediction of trends on how alloying additions affect the austenite-ferrite transformation. Further work in this regard should be focused on the interaction among different alloying elements. In addition, the proposed approach will have to be extended to austenite decomposition into complex microstructures that contain bainite and martensite in addition to ferrite.

\section{ACKNOWLEDGEMENTS}

The authors thank the Natural Sciences and Engineering Research Council (NSERC) of Canada and Evraz Inc. NA for financial support and Compute/Calcul Canada for computational resources. Furthermore, the authors would like to acknowledge I. Elfimov, H. Jin, H. Song, M. Greenwood, N. Ofori-Opoku, D. Panahi, H. van Landeghem, and T. Jia for their contributions to this work.

\section{REFERENCES}

1. J.R. Fekete, J.N. Hall, D.J. Muleman, and M. Rupp, Proceedings of the International Conference on New Development in Advanced High-Strength Sheet Steels (Warrendale, PA: AIST, 2008), p. 249.

2. Y. Funakawa, T. Shiozaki, K. Tomita, T. Yamamoto, and E. Maeda, ISIJ Int. 44, 1945 (2004).

3. A. Yoshie, M. Fujioka, Y. Watanabe, K. Nishioka, and H. Morikawa, ISIJ Int. 32, 395 (1992).

4. R. Shulkolsky, D.L. Rosburg, J.D. Chapman, and K.R. Barnes, Modeling, Control and Optimization in Nonferrous and Ferrous Industries, ed. F. Kongoli, B.G. Thomas, and K. Sawamiphakdi (Warrendale: TMS, 2003), p. 509.

5. A. Perlade, D. Grandemange, and T. Lung, Ironmak Steelmak 32, 299 (2005).

6. M. Militzer, ISIJ Int. 47, 1 (2007).
7. W.T. Geng, A.J. Freeman, and G.B. Olson, Phys. Rev. B 63, 165415 (2001).

8. A. Saha and G.B. Olson, J. Comput. Mater. Des. 14, 177 (2007).

9. Y. Mishin, M. Asta, and J. Li, Acta Mater. 58, 1117 (2010).

10. National Research Council (US). Committee on Integrated Computational Materials Engineering, Integrated Computational Materials Engineering: A Transformational Discipline for Improved Competitiveness and National Security (Washington, DC: The National Academic Press, 2008).

11. J.W. Cahn, Acta Metall. 10, 789 (1962).

12. M. Hillert, Acta Mater. 52, 5289 (2004).

13. G.R. Purdy and Y.J.M. Brechet, Acta Metall. Mater. 43, 3763 (1995).

14. G. Pariser, P. Shaffnit, I. Steinbach, and W. Bleck, Steel Res. 72,354 (2001).

15. M. Militzer, M.G. Mecozzi, J. Sietsma, and S. van der Zwaag, Acta Mater. 54, 3961 (2006).

16. F. Fazeli and M. Militzer, Metall. Mater. Trans. A 36A, 1396 (2005).

17. G. Kresse and J. Furthmüller, Phys. Rev. B 54, 11169 (1996).

18. G. Kresse and J. Furthmüller, Comput. Mater. Sci. 6, 15 (1996).

19. G. Kresse and D. Joubert, Phys. Rev. B 59, 1758 (1999).

20. H. Jin, I. Elfimov, and M. Militzer, J. Appl. Phys. 115, 093506 (2014).

21. T. Moriya, Prog. Theor. Phys. 33, 157 (1965).

22. P. Lejček, Grain Boundary Segregation in Metals (Berlin: Springer, 2010).

23. H. Song and J.J. Hoyt, Acta Mater. 60, 4328 (2012).

24. G.P. Krielaart and S. van der Zwaag, Mater. Sci. Technol. 14, 10 (1998)

25. H. Song and J.J. Hoyt, Acta Mater. 61, 1189 (2013).

26. B. Böttger, M. Apel, J. Eiken, P. Schaffnit, and I. Steinbach, Steel Res. Int. 79, 608 (2008).

27. M. Militzer, Curr. Opin. Solid State Mater. Sci. 15, 106 (2011).

28. D. Fan, S.P. Chen, and L.Q. Chen, J. Mater. Res. 14, 51 (1999).

29. S. Shahandeh, M. Greenwood, and M. Militzer, Model. Simul. Mater. Sci. Eng. 20, 065008 (2012).

30. M. Greenwood, J. Rottler, and N. Provatas, Phys. Rev. Lett. 105, 045702 (2010).

31. M. Greenwood, N. Ofori-Opoku, J. Rottler, and N. Provatas, Phys. Rev. B. 84, 064104 (2011).

32. M. Greenwood, C.W. Sinclair, and M. Militzer, Acta Mater. $60,5752(2012)$.

33. N. Ofori-Opoku, J. Stolle, Z.F. Huang, and N. Provatas, Phys. Rev. B 88, 104106 (2013).

34. D. Panahi, Y. Bai, H.S. Zurob, G.R. Purdy, C.R. Hutchinson, and Y. Brechet, Solid State Phenom. 172-174, 539 (2011).

35. C. Qiu, H.S. Zurob, D. Panahi, Y.J.M. Brechet, G.R. Purdy, and C.R. Hutchinson, Metall. Mater. Trans. A 44A, 3472 (2013).

36. H.S. Zurob, D. Panahi, C.R. Hutchinson, Y. Brechet, and G.R. Purdy, Metall. Mater. Trans. A 44A, 3456 (2013).

37. T. Jia and M. Militzer, ISIJ Int. 52, 644 (2012).

38. M. Enomoto, C.L. White, and H.I. Aaronson, Metall. Trans. A 19A, 1807 (1988).

39. M. Guttmann, Surf. Sci. 53, 213 (1975). 